\title{
Increased $\mathrm{Ca}^{2+}$ influx through $\mathrm{Ca}_{\mathrm{v}} 1.2$ drives aortic valve calcification
}

\author{
Maiko Matsui, ${ }^{1}$ Rihab Bouchareb, ${ }^{2}$ Mara Storto, ${ }^{1}$ Yasin Hussain, ${ }^{1}$ Andrew Gregg, ${ }^{1}$ Steven O. Marx, ${ }^{3}$ \\ and Geoffrey S. Pitt ${ }^{1,2}$ \\ ${ }^{1}$ Cardiovascular Research Institute, Weill Cornell Medicine, New York, New York, USA. ${ }^{2}$ Zena and Michael A. Wiener \\ Cardiovascular Institute, Icahn School of Medicine at Mount Sinai, New York, New York, USA. ${ }^{3}$ Division of Cardiology, \\ Department of Medicine, and Department of Pharmacology, Vagelos College of Physicians and Surgeons, Columbia \\ University, New York, New York, USA.
}

Calcific aortic valve disease (CAVD) is heritable, as revealed by recent CWAS. While polymorphisms linked to increased expression of CACNA1C - encoding the Ca 1.2 L-type voltage-gated $\mathrm{Ca}^{2+}$ channel - and increased $\mathrm{Ca}^{2+}$ signaling are associated with CAVD, whether increased $\mathrm{Ca}^{2+}$ influx through the druggable $\mathrm{Ca}_{\mathrm{v}} 1.2$ causes CAVD is unknown. We confirmed the association between increased $\mathrm{Ca}_{\mathrm{v}} 1.2$ expression and CAVD in surgically removed aortic valves from patients. We extended our studies with a transgenic mouse model that mimics increased $\mathrm{Ca}_{\mathrm{v}} 1.2$ expression within aortic valve interstitial cells (VICs). In young mice maintained on normal chow, we observed dystrophic valve lesions that mimic changes found in presymptomatic CAVD and showed activation of chondrogenic and osteogenic transcriptional regulators within these valve lesions. Chronic administration of verapamil, a Ca 1.2 antagonist used clinically, slowed the progression of lesion development in vivo. Exploiting VIC cultures, we demonstrated that increased $\mathrm{Ca}^{2+}$ influx through $\mathrm{Ca}_{\mathrm{v}} 1.2$ drives signaling programs that lead to myofibroblast activation of VICs and upregulation of genes associated with aortic valve calcification. Our data support a causal role for $\mathrm{Ca}^{2+}$ influx through $\mathrm{Ca}_{\mathrm{v}} 1.2$ in CAVD and suggest that early treatment with $\mathrm{Ca}^{2+}$ channel blockers is an effective therapeutic strategy.

Conflict of interest: The authors have declared that no conflict of interest exists.

Copyright: (c) 2022, Matsui et al. This is an open access article published under the terms of the Creative Commons Attribution 4.0 International License.

Submitted: October 5, 2021 Accepted: January 28, 2022 Published: March 8, 2022

Reference information: /CI Insight. 2022;7(5):e155569. https://doi.org/10.1172/jici. insight.155569.

\section{Introduction}

Calcific aortic valve disease (CAVD) is a life-threatening disorder affecting approximately $2 \%$ of people older than 65 years. Excess calcium phosphate deposition in the aortic valve stiffens the tissue and ultimately narrows the valve orifice. The consequent increase in left ventricular pressure strains the left ventricle. Common clinical outcomes, if this condition is untreated, are angina, heart failure, syncope, and/or sudden death. Surgical or transcatheter aortic valve replacement are the only current therapeutic options (1); no drug treatments have been shown to reverse or slow progression (2).

While the underlying mechanism of CAVD was previously thought to be wear and tear, researchers subsequently showed that calcification initiates after injury-mediated or inflammatory-mediated disruption of the valve endothelial surface, which leads to 2 different but overlapping responses, both of which start with differentiation of fibroblast-like valve interstitial cells (VICs) that reside below the endothelial surfaces (3). First, dystrophic calcification results from myofibroblast differentiation of VICs in response to paracrine action of TGF- $\beta$. Identifying features of VICs differentiated into myofibroblasts are expression of $\alpha$-smooth muscle actin ( $\alpha$-SMA), which myofibroblasts organize into bundles or stress fibers, and that VICs become contractile. Activated myofibroblasts remodel the extracellular matrix that alters the local milieu in ways that promote calcification, such as by secretion of MMPs that release latent TGF- $\beta$ within the extracellular matrix, thereby augmenting VIC differentiation to myofibroblasts. Activated myofibroblasts eventually undergo apoptosis and consequently deposit calcium salts that precipitate calcification (4). The second process by which VICs contribute to calcification is by undergoing osteogenesis. The ectopically transformed osteoblasts secrete bone matrix $(5,6)$, as reflected by upregulation of canonical osteogenic signaling pathways, such as the BMP pathway and the Wnt-signaling pathway, in diseased calcific aortic valves (6-8). Likewise, chondrogenic signaling in diseased valves is marked by the expression of Aggrecan $(A C A N)$ and SOX9, a major chondrogenic transcriptional regulator (9). 
Attempts at medical therapy to slow or ameliorate the development of CAVD have been unsuccessful, and failures have been attributed to the late initiation of treatment, when calcification is present and thus difficult to halt or reverse. Hence, while understanding the early events that eventually result in CAVD might provide opportunities to intervene in a timely manner, the initial events that activate VICs to cause dystrophic calcification or undergo osteogenesis are not well understood. Moreover, although the pathophysiological consequences of CAVD result primarily from stiffened valve leaflets, a recent study of donor heart valves from individuals not known to have aortic valve disease showed that nascent disease is characterized by intrinsic calcification at the hinge regions rather than in the leaflets (10). Focusing on these changes in the hinge regions, therefore, may offer a window of opportunity for therapeutic intervention.

One approach to identify the early changes is to focus on genetic susceptibility, because genetics represents factors continuously present and starting from birth. Indeed, GWAS have suggested genetic susceptibility to CAVD, but whether any of these associated loci are causal and the mechanisms by which variants increase susceptibility are largely unknown. An SNP near LPA demonstrated genome-wide significance (11), consistent with previous studies demonstrating an association between levels of the $L P A$-encoded lipoprotein(a) and aortic valve calcification (12-15). SNPs near PALMD, IL6, ALPL, and the noncoding TEX41 also associate with aortic valve calcification (16-18), but whether variants at these loci are causal is unknown, as are the molecular mechanisms underlying disease association. A meta-analysis of 2 GWAS suggested an association between increased expression of $C A C N A 1 C$, which encodes the $\mathrm{Ca}_{\mathrm{v}} 1.2 \mathrm{~L}$-type voltage gated $\mathrm{Ca}^{2+}$ channel, and CAVD (19). That association was supported by several additional observations, including increased $C A C N A 1 C$ transcripts in calcified versus noncalcified valves and a gene-set enrichment analysis of all SNPs (not only those within $C A C N A 1 C$ ) with moderate $(P<0.0001)$ disease association that identified the calcium-signaling pathway (Kyoto Encyclopedia of Genes and Genomes identifier hsa04020) as the top gene set.

$\mathrm{Ca}_{\mathrm{v}} 1.2$ is the predominant voltage-gated $\mathrm{Ca}^{2+}$ channel in the myocardium, in which $\mathrm{Ca}_{\mathrm{v}} 1.2$ initiates excitation-contraction coupling. Additionally, $\mathrm{Ca}_{\mathrm{v}} 1.2$ serves essential roles in other excitable tissues, such as the brain and endocrine tissue, and in vascular smooth muscle cells, where it is the target of commonly prescribed $\mathrm{Ca}^{2+}$ channel blocker antihypertension drugs. Expression in cardiac valves had not been previously described, so a role in CAVD was unexpected. An increasing amount of evidence, however, suggests that $\mathrm{Ca}_{\mathrm{v}} 1.2$ exerts roles in nonexcitable tissues (20) and, as a plasma membrane voltage-gated $\mathrm{Ca}^{2+}$ channel, $\mathrm{Ca}_{\mathrm{v}} 1.2$ sits at the pinnacle of myriad intracellular $\mathrm{Ca}^{2+}$-dependent signaling events capable of triggering myofibroblast activation and osteogenic transformation. If increased $\mathrm{Ca}^{2+}$ influx through $\mathrm{Ca}_{\mathrm{v}} 1.2$ is causal for calcific aortic stenosis, then readily available FDA-approved drugs would be lead candidates for therapeutic intervention.

With human aortic valve samples, we confirmed increased $\mathrm{Ca}_{\mathrm{v}} 1.2$ in valves surgically excised for calcific aortic stenosis, compared with noncalcified valves. We then generated a mouse model to mimic increased $\mathrm{Ca}_{\mathrm{v}} 1.2$ expression within the aortic valve, with which we observed accelerated aortic valve calcification in vivo, thus showing that increased $\mathrm{Ca}_{\mathrm{v}} 1.2$ expression is, indeed, causal. Chronic administration of verapamil, a $\mathrm{Ca}_{\mathrm{v}} 1.2$ antagonist used clinically, slowed valve calcification, suggesting that reduction of $\mathrm{Ca}^{2+}$ influx through $\mathrm{Ca}_{\mathrm{v}} 1.2$ is a therapeutic opportunity. Using transcriptional profiling in cultured mouse VICs, we then defined how increased $\mathrm{Ca}^{2+}$ influx through $\mathrm{Ca}_{\mathrm{v}} 1.2$ could activate downstream signaling pathways capable of driving valve calcification.

\section{Results}

The researchers who conducted the GWAS that identified an association between $C A C N A 1 C$ and CAVD reported increased $C A C N A 1 C$ and $R U N X 2$ transcripts within calcified versus noncalcified human aortic valves (19). Using IHC, we found that calcified areas of valves obtained from individuals with CAVD valves also had increased levels of $\mathrm{Ca}_{\mathrm{v}} 1.2$ protein compared with valves obtained from individuals without CAVD (Figure 1A). Moreover, the increased signal for $\mathrm{Ca}_{\mathrm{v}} 1.2$ protein in valves obtained from individuals with CAVD was most prominent in areas that also displayed a strong signal for the osteogenic marker, RUNX2. In contrast, only a weak $\mathrm{Ca}_{\mathrm{v}} 1.2$ or RUNX2 signal was observed in valves excised from hearts harvested for transplant and without CAVD (Figure 1B). Three CAVD valves and 3 valves without CAVD were tested in toto with similar results (Figure 1, C and D).

To test if increased $\mathrm{Ca}_{\mathrm{v}} 1.2$ expression within the aortic valve is causal for valve calcification, we generated a mouse model in which transgenic $\mathrm{Ca}_{\mathrm{v}} 1.2$ expression was specifically increased in the valve tissue. 

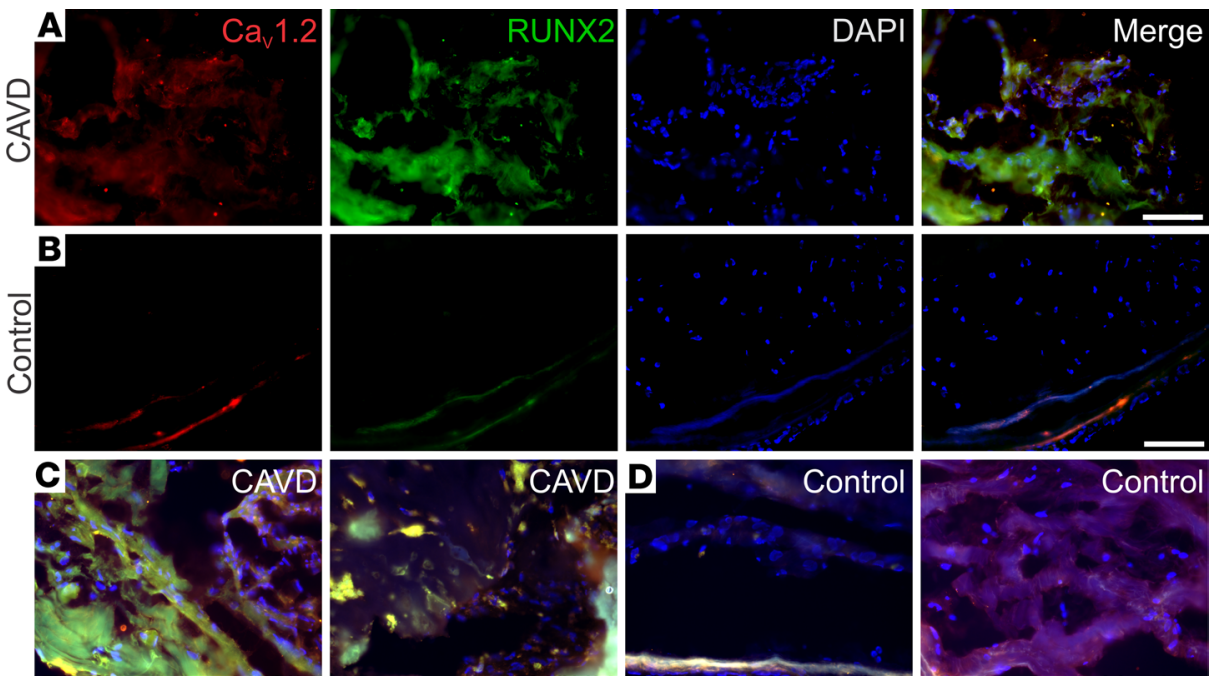

Figure 1. Increased $\mathrm{Ca}_{\mathrm{v}} 1.2$ and RUNX2 expression in calcified segments of aortic valves from individuals with CAVD. ( $A$ and $\mathbf{B}$ ) IHC analysis of $C a_{v} 1.2$ (red) and RUNX2 (green) of valve tissue excised from a patient with CAVD (A) or a valve excised from a heart harvested for transplant and without CAVD (B). The blue signal is DAPI, and merged images are shown in the right-most panels for each. (C and D) Merged images for 2 additional valves from patients with CAVD (C) and from valves excised from hearts harvested for transplant and without CAVD (D). Scale bars: $100 \mu \mathrm{m}$.

We used a model in which transgenic $\mathrm{Ca}_{\mathrm{v}} 1.2$ expression was targeted specifically to the aortic valve, modifying a strategy we have used previously to target $\mathrm{Ca}_{\mathrm{v}} 1.2$ expression to the mandible (21) or osteoblasts (22, 23). Here, we crossed a $S c x$-Cre driver mouse with mice with a $\mathrm{Ca}_{\mathrm{v}} 1.2 \mathrm{WT} c \mathrm{CNA}\left(\mathrm{Ca}_{\mathrm{v}} 1.2^{\mathrm{WT}}\right)$ inserted into Rosa26 and downstream of a floxed STOP codon (Figure 2A). Scx-Cre drives expression in heart valves but not in the myocardium $(24,25)$, as we confirmed with a GFP reporter (Figure 2B). Thus, these $S c x-C a_{V} 1.2^{W T}$ mice express transgenic $\mathrm{Ca}_{\mathrm{v}} 1.2$ specifically in the aortic valve. As we previously showed, this transgenic $\mathrm{Ca}_{\mathrm{v}} 1.2$ expression model increases $\mathrm{Ca}^{2+}$ influx (21) and thereby provides a model that mimics the increased $\mathrm{Ca}_{\mathrm{v}} 1.2$ transcription and protein levels observed specifically in the aortic valves of patients with CAVD (19) and the increased $\mathrm{Ca}_{\mathrm{v}} 1.2$ expression that we detected in a human aortic valve sample (Figure 1A).

Histological analyses with pentachrome staining of transgenic $S c x-C a_{V} 1.2^{W T}$ valves obtained from 4-month-old mice showed the presence of discrete lesions in all valves examined (Figure 2C), but in only 1 valve from a Cre-negative control mouse (for Cre-negative control, $n=12$; for $S c x-C a_{V} 1.2^{W T}, n=10$; Fisher exact test, $P<0.0001$ ). Thus, increased $\mathrm{Ca}_{\mathrm{v}} 1.2^{\mathrm{WT}}$ expression in the aortic valve was sufficient to cause development of calcified lesions. Also remarkable was that these lesions were present in 4-month-old mice, a stage much earlier than in typical murine CAVD models. Consistent with the relatively young age of the mice at which these lesions appeared, the lesions were predominantly located in the hinge regions rather than the valve leaflets, reflecting nascent disease seen in humans (10). Notably, the mice were maintained on a normal chow diet, in contrast to a high cholesterol diet commonly used in murine models of CAVD, and thus these early lesions do not require elevated lipid levels that are a CAVD risk factor.

We then developed a model to increase $\mathrm{Ca}^{2+}$ influx through $\mathrm{Ca}_{\mathrm{v}} 1.2$ to accelerate the disease process in the mouse model. We exploited a gain-of-function mutant $\mathrm{G} 406 \mathrm{R}$ mutation $\left(\mathrm{Ca}_{\mathrm{v}} 1.2^{\mathrm{TS}}\right)$ found in individuals with the multisystem disorder Timothy syndrome (26) that disrupts $\mathrm{Ca}_{\mathrm{v}} 1.2$ channel voltage-dependent inactivation and thereby promotes increased $\mathrm{Ca}^{2+}$ through the channel. Using the Rosa26 knock-in strategy, but with the gain-of-function mutant $\mathrm{Ca}_{\mathrm{v}} 1.2^{\mathrm{TS}}$ expressed rather than the $\mathrm{Ca}_{\mathrm{v}} 1.2^{\mathrm{WT}}$ channel, we examined whether targeted expression in the aortic valve with $S c x$-Cre accelerated the appearance of calcified valve lesions. Figure $2 \mathrm{D}$ shows heterogeneous histological evidence of lesions in the valves from the $S c x-C a_{V} 1.2^{T S}$ mice. We observed lesions in all $(n=22) S c x-C a_{V} 1.2^{T S}$ aortic valves examined but in only 1 valve from 12 Cre-negative control mice (Fisher exact test, $P<0.0001$ ). Alizarin red and von Kossa staining demonstrated calcification within the lesions, but not in the adjacent histologically normal-appearing valve (Figure $2 \mathrm{E}$ ), again consistent with the histological appearance and using the same detection methods as for the nascent lesions seen in humans (10). Thus, valve-specific expression of a gain-of-function mutant $\mathrm{Ca}_{\mathrm{v}} 1.2^{\mathrm{TS}}$ exacerbated the development of calcified lesions and provides a model to accelerate the development of aortic valve calcification. 

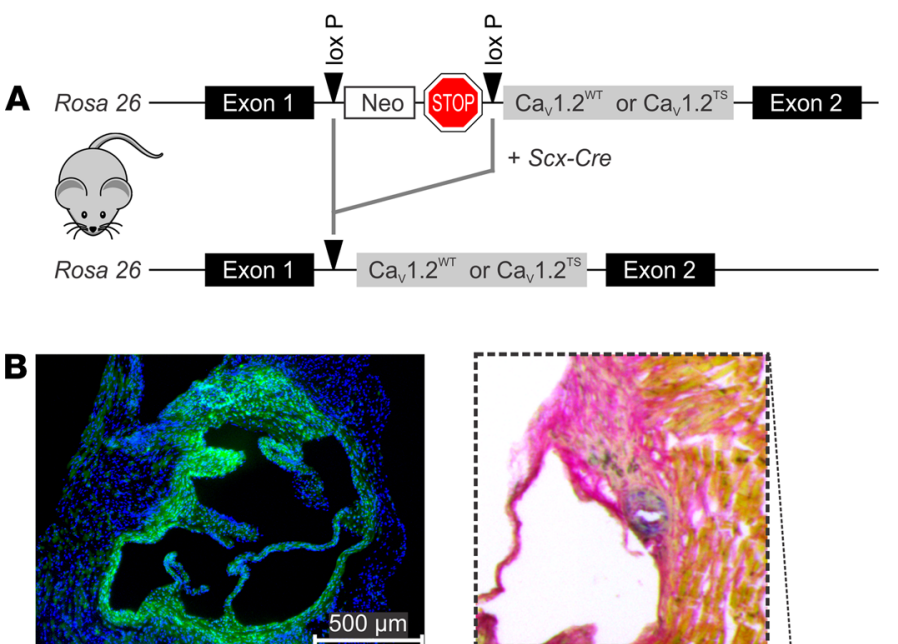

ScX-Cre;R26R GFP/t

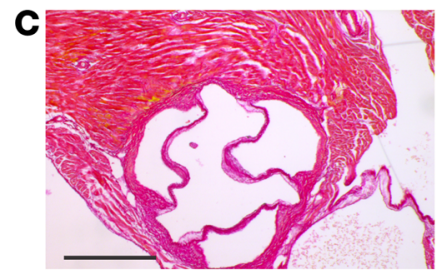

Cre-

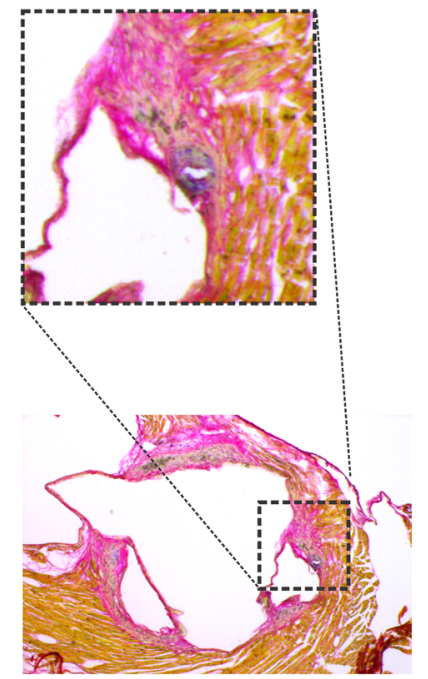

$\operatorname{Scx}-\mathrm{Ca}_{v} 1.2^{\mathrm{WT}}$
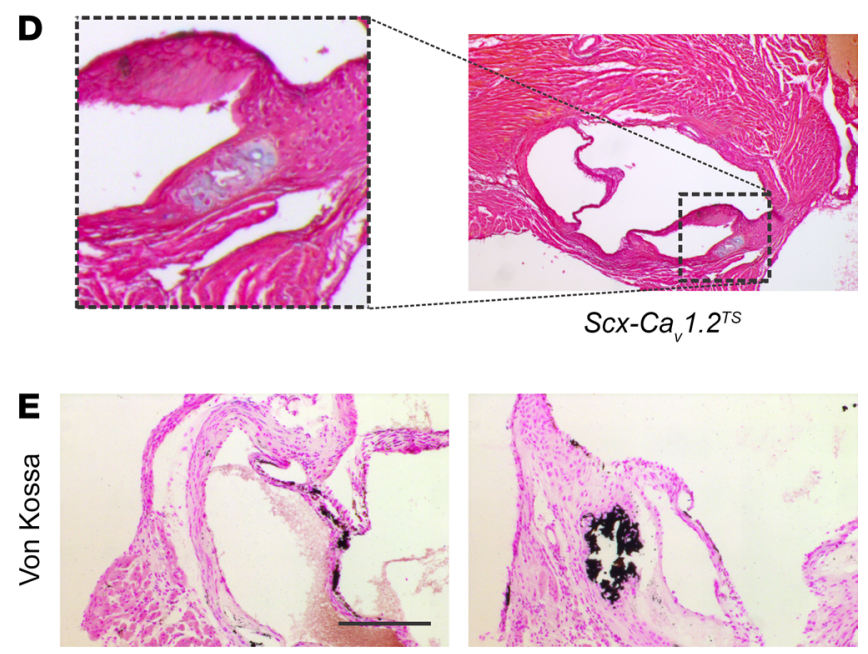

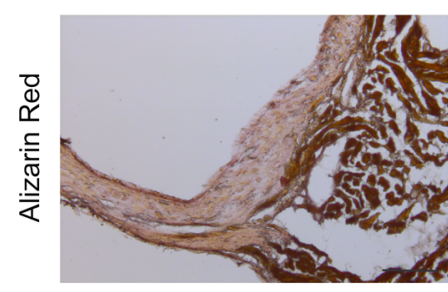

Cre-

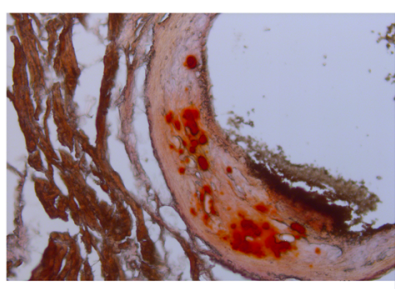

$\mathrm{Scx}-\mathrm{Ca}_{v} 1.2^{\mathrm{TS}}$

Figure 2. Increased $\mathrm{Ca}_{\mathrm{v}}$ 1. 2 expression in the aortic valve is causal for CAVD. (A) Transgenic strategy for inducible Ca 1.2 expression. (B) GFP immunofluorescence (DAPI counterstain) showing aortic valve targeting with Scx-Cre. (C and D) Pentachrome staining shows lesions (inset) in Ca ${ }_{v}{ }^{1} 2^{\text {WT }}$ (C) and $\mathrm{Ca}_{\mathrm{v}} 1.2^{\mathrm{TS}}$ (D) but not in $\mathrm{Cre}^{-}$control mice (C). Scale bar: $200 \mu \mathrm{m}$. (E) Von Kossa and alizarin red stains demonstrate calcification of valve lesions in mice overexpressing $C a_{\mathrm{v}} 1.2^{\mathrm{TS}}$ in the valve. Scale bar: $100 \mu \mathrm{m}$.

Further characterization of the lesions with immunohistochemical markers revealed discrete dystrophic activation. For example, Figure 3A demonstrates increased $\alpha$-SMA specifically within the discrete valve lesions in $S c x-C a_{V} 1.2^{T S}$ (and not the $C r e^{-}$control mice), but not in the unaffected adjacent valve tissue. Moreover, the lesions shown in Figure 3, B and C, display features of chondrogenesis: Sox 9 and aggrecan (Acan) were both detected in the lesions in $S c x-C a_{V} 1.2^{T S}$ mice but not in the unaffected valve areas. This finding is consistent with the recent discovery that Sox9 and Acan are upregulated downstream of $\mathrm{Ca}^{2+}$ influx through $\mathrm{Ca}_{\mathrm{v}} 1.2$ in developing bone (27). Early features of osteogenesis, as indicated by the expression of the master osteogenic transcriptional factor Runx2, were also detected (Figure 3D).

Because transgenic expression of $\mathrm{Ca}_{\mathrm{v}} 1.2$, mimicking the increased expression of $\mathrm{Ca}_{\mathrm{v}} 1.2$ associated with CAVD in patients (19), led to the appearance of valve lesions and activation of the dystrophic and osteogenic signaling pathways, we next tested whether administrating a $\mathrm{Ca}^{2+}$ channel blocker (verapamil, $10 \mathrm{mg} / \mathrm{kg} / \mathrm{d}$ ) in vivo via a mini-osmotic pump reduced the appearance of the dystrophic lesions in the $S c x-C a_{V} 1.2^{T S}$ model. We chose verapamil over a dihydropyridine because the Rosa26 $\mathrm{Ca}_{\mathrm{v}} 1.2^{\mathrm{TS}}$ model has an engineered mutation that renders the channels insensitive to dihydropyridines (28). Mini-osmotic pumps containing verapamil or vehicle control were implanted into 2-month-old $S c x-C a_{V} 1.2^{T S}$ mice and lesion area was quantified in valves isolated 28 days later. Lesion area was significantly reduced in verapamil-treated mice $(n=16 ; P<0.05)$ compared with vehicle-treated control mice (Figure 4$)$. Thus, in a model of increased $\mathrm{Ca}_{\mathrm{v}} 1.2$ expression in the valve, chronic blockade of $\mathrm{Ca}^{2+}$ influx effectively reduced valve lesions.

To explore signaling pathways downstream of $\mathrm{Ca}^{2+}$ influx via $\mathrm{Ca}_{\mathrm{v}} 1.2$ that contributed to valve calcification, we used a mouse VIC culture system. We first confirmed that our VIC culture preparations were free of valve endothelial cell contamination by performing quantitative PCR (qPCR) for endothelial cell and VIC marker transcripts. Figure 5A shows expression levels of VIC-specific transcripts $\alpha$-SMA (Acta2) and prolyl-4-hydrolase (P4ha1) that are more than 3000-fold to 60,000-fold higher than expression levels of endothelial cell markers $C D 31$ or von Willebrand's factor $\left(\Delta C_{t}\right.$ range, 11.6-15.9). 

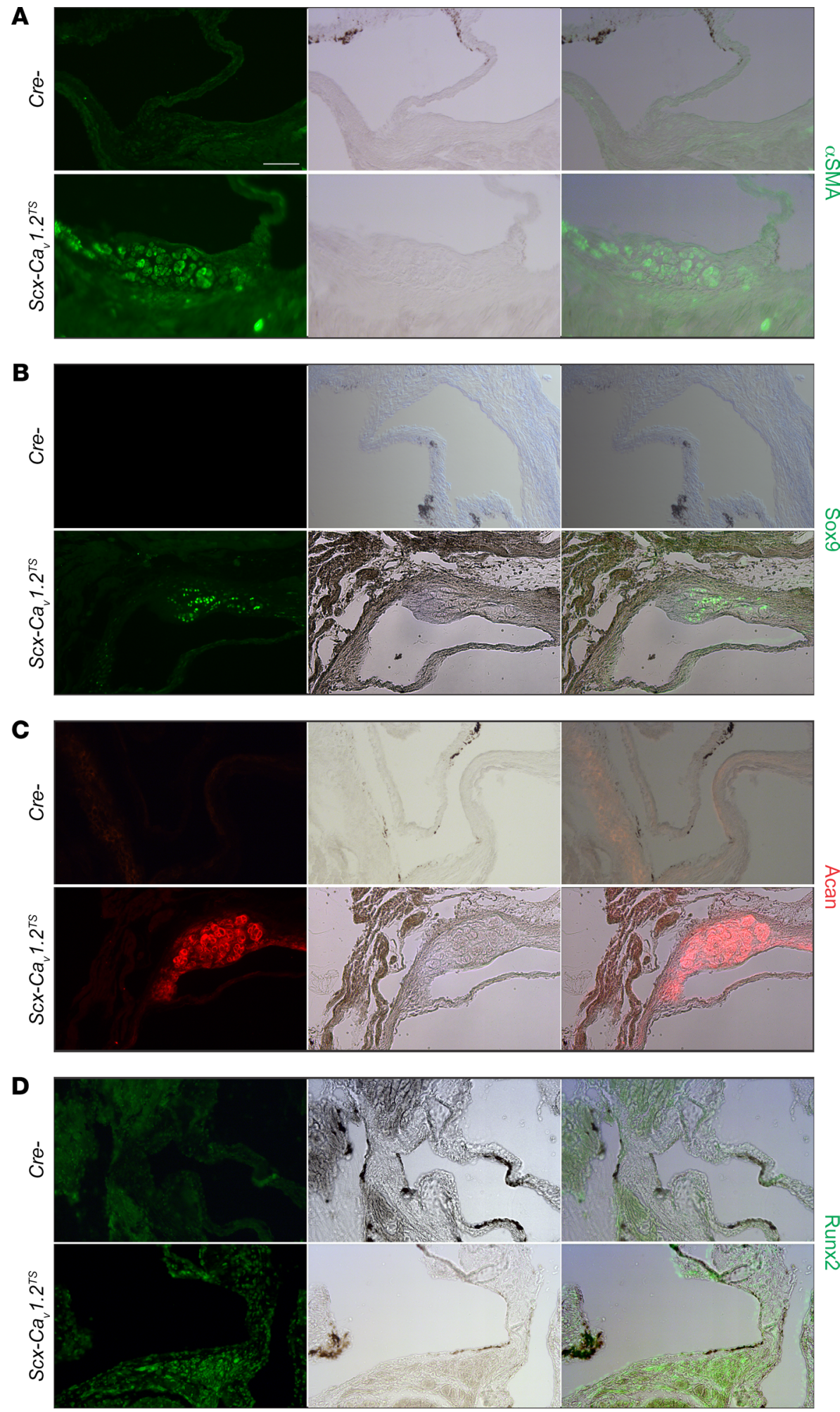

Figure 3. Lesion-specific upregulation of chondrogenic and osteogenic makers in vivo. Immunofluorescence for $\alpha$-SMA (A), Sox9 (B), Aggrecan (C), and Runx2 (D) with accompanying light transmission and merged images of valves from $C r e^{-}$control and $S c x-C a_{v} 1.2^{\text {TS }}$ mice shows expression of the specific marker within the valve lesion but not in the unaffected valve. For each marker, examples from $\geq 3$ samples. Scale bar: $100 \mu \mathrm{m}$. 
A

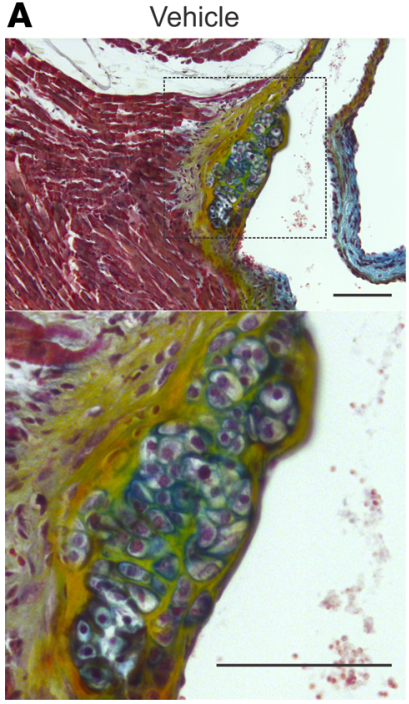

Verapamil $10 \mathrm{mg} / \mathrm{kg} / \mathrm{day}$

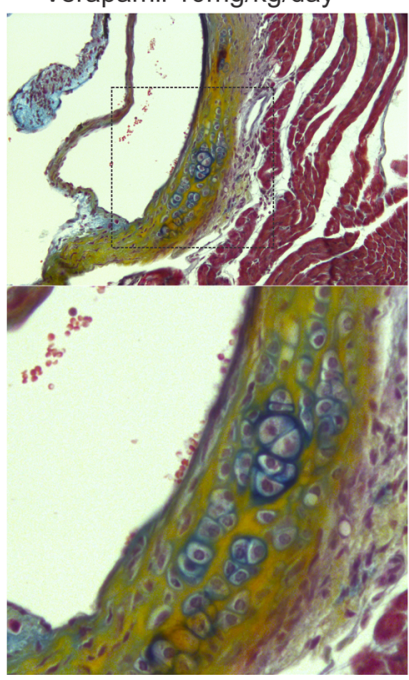

B

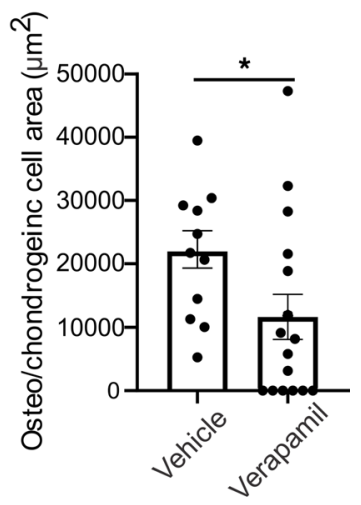

Figure 4. Blockade of $\mathrm{Ca}^{2+}$ influx through $\mathrm{Ca}_{v} \mathbf{1 . 2}$ reduces aortic valve lesions in $\mathbf{S c x}-\mathrm{Ca}_{v}{ }^{1.2^{T S}}$ mice. (A) Pentachrome stain of aortic valves from 3-month-old $S c x-C a_{v} 1.2^{T S}$ mice. Valves were isolated after 4 weeks of treatment by mini-osmotic pumps with verapamil or vehicle control. Inset shows magnification of lesion. (B) Quantification of lesion area. ${ }^{*} P=0.04$ (2-sided $t$ test). Scale bars: $100 \mu \mathrm{m}$. Osteo, osteogenic.

Because the human GWAS showed a correlation between increased $\mathrm{Ca}_{\mathrm{v}} 1.2$ expression in the valve and valve calcification, and our mouse models demonstrated that increased $\mathrm{Ca}_{\mathrm{v}} 1.2$ expression in the valve is casual for valve calcification, we tested if increased $\mathrm{Ca}_{\mathrm{v}} 1.2$ expression in cultured VICs is sufficient to activate dystrophic signaling pathways. As a first test, we cultured VICs obtained from the mice in which $C a_{V} 1.2^{W T}$ was inserted into the Rosa26 locus downstream of a floxed STOP codon (see Figure 2A) and activated the $C a_{V} 1.2^{W T}$ transgene by infecting the cells with an adenovirus expressing Cre recombinase (to remove the STOP codon) or an adenovirus expressing GFP (as a control). We then performed qPCR and immunocytochemistry for $\alpha$-SMA, an indicator of myofibroblast differentiation, which showed that enhanced expression of Cacnalc in VICs increased $\alpha$-SMA by approximately $40 \% 2$ days after infection (Figure 5B) and led to the appearance of $\alpha$-SMA stress fibers that were not present after control GFP infection (Figure $5 \mathrm{C}$ ), thus providing initial mechanistic insight into the downstream effects of increased $\mathrm{Ca}_{\mathrm{v}} 1.2$ expression in VICs. We confirmed that the observed myofibroblast activation was not an artifact of Cre infection: qPCR of RNA from cultured VICs isolated from WT mice infected with GFP or Cre showed no difference in $\alpha$-SMA expression levels (Figure 5D). As with the in vivo experiments, we chose verapamil over a dihydropyridine because the $\mathrm{Ca}_{\mathrm{v}} 1.2^{\mathrm{TS}}$ model has an engineered mutation that renders the channels insensitive to dihydropyridines (28).

Finally, we hypothesized that the additional $\mathrm{Ca}^{2+}$ influx through the $\mathrm{Ca}_{\mathrm{v}} 1.2^{\mathrm{TS}}$ mutant channel would generate an even larger increase in $\alpha$-SMA expression. We infected VICs obtained from mice expressing the floxed-STOP $C a_{V} 1.2^{T S}$ transgene knocked into Rosa26 (Figure 2B) with either adenovirus expressing Cre recombinase (to remove the STOP codon) or GFP (control) and measured the expression of $\alpha$-SMA by quantitative RT-PCR 2 days after infection. In comparison with overexpression of the $\mathrm{Ca}_{\mathrm{v}} 1.2^{\mathrm{WT}}$ channel and the resultant 1.4-fold increase in $\alpha$-SMA (Figure 5B), expression of the gain-of-function mutant $\mathrm{Ca}_{\mathrm{v}} 1.2^{\mathrm{TS}}$ channel generated an approximately 2-fold increase in $\alpha$-SMA expression (Figure 5E), further augmenting $\mathrm{Ca}^{2+}$ influx with the $\mathrm{Ca}_{\mathrm{v}} 1.2^{\mathrm{TS}}$ channel amplified the activation of $\alpha$-SMA. Additionally, we confirmed that the resultant increase in $\alpha$-SMA after expression of $\mathrm{Ca}_{\mathrm{v}} 1.2$ derived from $\mathrm{Ca}^{2+}$ influx through the transgenic $\mathrm{Ca}_{\mathrm{v}} 1.2$ channels, because blockade with verapamil eliminated any increase in $\alpha$-SMA expression (Figure $5 \mathrm{~F}$ ).

We next performed bulk RNA-Seq to query the initial downstream signaling pathways and factors affected by increased $\mathrm{Ca}^{2+}$ influx through $\mathrm{Ca}_{\mathrm{v}} 1.2$ in VICs. Because the gain-of-function mutant $\mathrm{Ca}_{\mathrm{v}} 1.2^{\mathrm{TS}}$ channel amplified the $\alpha$-SMA response in cultured VICs, we exploited the $\mathrm{Ca}_{\mathrm{v}} 1.2^{\mathrm{TS}}$ channel for the RNASeq experiments. We treated cultured VICs isolated from $\mathrm{Ca}_{\mathrm{v}} 1.2^{\mathrm{TS}}$ mice, plated them on hard plastic with adenovirus expressing Cre recombinase or GFP (control), and isolated RNA for bulk RNA-Seq 48 hours 
A

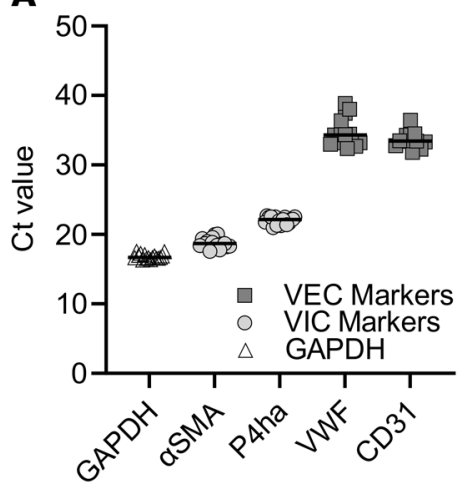

D

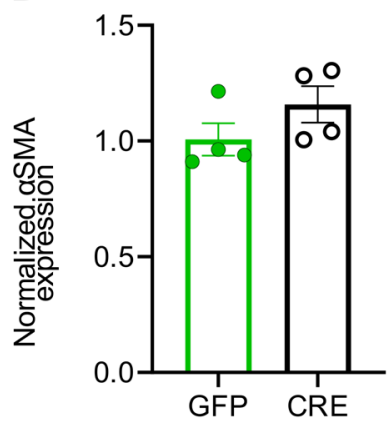

B

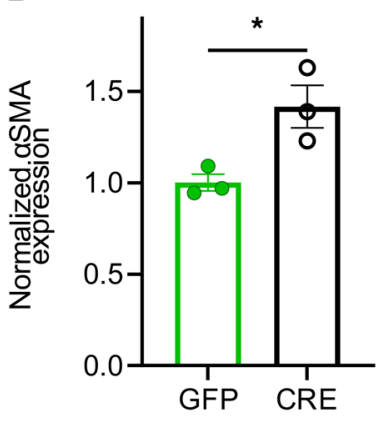

$\mathbf{E}$

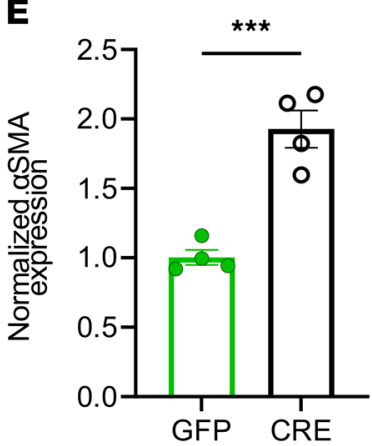

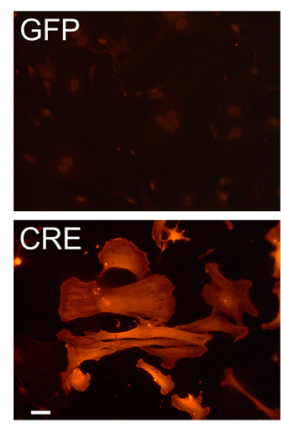

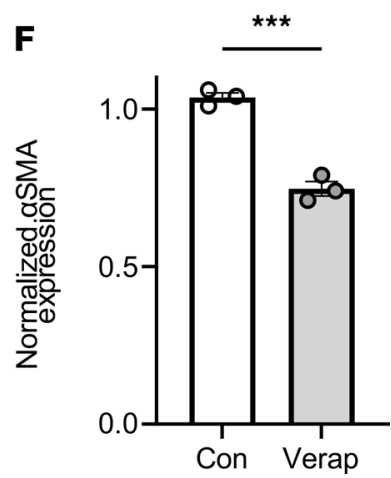

Figure 5. Blockade of $\mathrm{Ca}^{2+}$ influx through $\mathrm{Ca}_{\mathrm{v}} \mathbf{1 . 2}$ reduces aortic valve lesions in Scx-Ca $\mathbf{1} . \mathbf{2}^{\mathrm{TS}}$ mice. (A) qPCR showing expression of VIC markers ( $\alpha$-SMA and P4ha) compared with endothelial cell markers (VWF and CD31) in cultured VICs. (B) Normalized (to GAPDH) $\alpha$-SMA expression after 48 hours in $\mathrm{Ca}_{\mathrm{v}} 1.2^{\mathrm{WT}}$-expressing VICs (after infection with virus expression GFP or Cre recombinase). ${ }^{*} P<0.05$. (C) Anti- $\alpha$-SMA immunofluorescence in Ca $1.2^{\mathrm{TS}}$ VIC cultures expressing Cre or GFP. Scale bar: $50 \mu \mathrm{m}$. (D) Normalized (to GAPDH) $\alpha$-SMA expression after 48 hours in WT VICs (after infection with virus expression GFP or Cre recombinase). $P=$ NS. (E) Normalized (to GAPDH) $\alpha$-SMA expression after 48 hours in $\mathrm{Ca}_{\mathrm{v}} 12^{\mathrm{TS}}$ expressing VICs (after infection with virus expression GFP or Cre recombinase). $P<0.001$. (F) qPCR shows verapamil decreases relative expression of $\alpha-S M A$ in $C a_{v} 1.2^{\text {TS }}$ expressing VICs. ${ }^{* * *} P<0.001$. (B-F) Statistical comparisons were performed with 2-sided $t$ tests. Con, control; VEC, valve endothelial cell.

( $n=4$ mice each) and 72 hours ( $n=3$ mice each) after infection. Principal component analysis showed excellent separation of Cre-infected versus GFP-infected samples (Supplemental Figure 1, A and B; supplemental material available online with this article; https://doi.org/10.1172/jci.insight.155569DS1). In the 48-hour experiment, we observed upregulation of $\alpha$-SMA 2.36-fold \pm 0.12 -fold $(n=4$ each; adjusted $P\left[P_{a d j}\right]<1 \times 10^{-59}$ ), similar to what we observed by qPCR (Figure 5E). Between the Cre or GFP infection samples, we observed 10,540 protein-coding genes differentially expressed $\left(P_{a d j}<0.05\right)$, of which 5193 were up-regulated and 5347 were downregulated (Figure 6, A and B). A heat map of the 2724 transcripts for which expression in the Cre infected VICs was upregulated or downregulated by more than 2-fold is shown in Figure 6C.

We performed a separate analysis on RNA isolated 72 hours after Cre or GFP infection ( $n=3$ each), which revealed 10,306 differentially expressed protein-coding genes, of which 5118 were upregulated and 5188 were downregulated (Supplemental Figure 1, C and D). There were 8908 differentially expressed genes common to both data sets and there was a tight correlation $\left(r^{2}=0.86\right)$ between the $\log _{2}$-fold changes in expression values (Supplemental Figure 1E), suggesting limited overall differences in gene expression after the additional 24-hour exposure to increased $\mathrm{Ca}_{\mathrm{v}} 1.2$ expression. Therefore, to highlight the initial consequences of $\mathrm{Ca}^{2+}$-dependent signaling downstream of $\mathrm{Ca}_{\mathrm{v}} 1.2$ in the VICs, we focused our analyses on the data set obtained 48 hours after viral infection.

Gene ontology analyses by ingenuity pathway analysis (IPA) revealed that the top gene set among upregulated transcripts was the calcium-signaling pathway $\left(P_{a d j}=4.38 \times 10^{-13}\right)$ (Figure 6D), mirroring the top gene set for all SNPs that had moderate association with calcified human aortic valves (19) and consistent with upregulation of $\mathrm{Ca}^{2+}$ signaling by activating $\mathrm{Ca}_{\mathrm{v}} 1.2^{\mathrm{TS}}$ expression. A top upregulated (68.0-fold \pm 16.6 -fold; $\left.P_{a d j}=2.40 \times 10^{-53}\right) \mathrm{Ca}^{2+}$-signaling gene was $\mathrm{Nfatc2}$, a transcription factor activated by the $\mathrm{Ca}^{2+}$-sensitive phosphatase calcineurin. The next gene set identified by IPA was the hepatic 
A
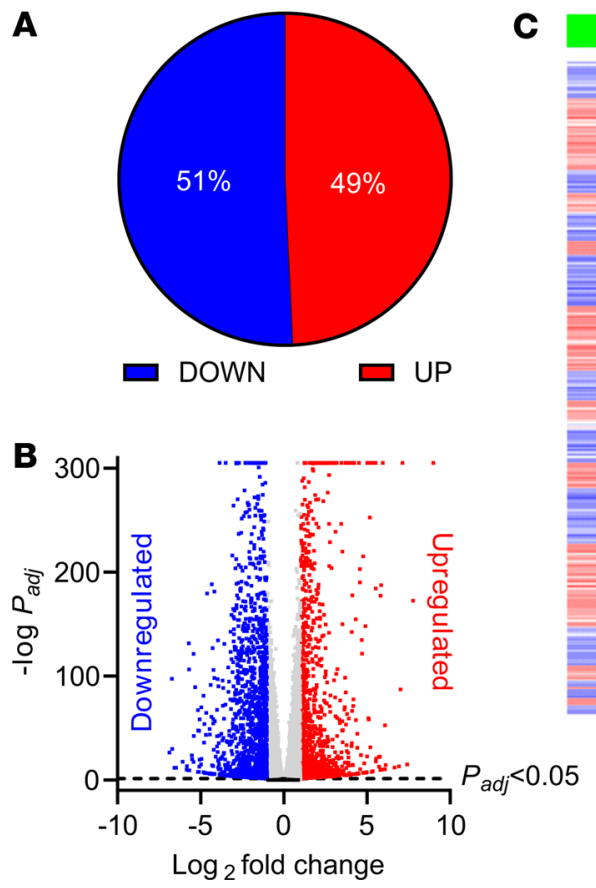

C

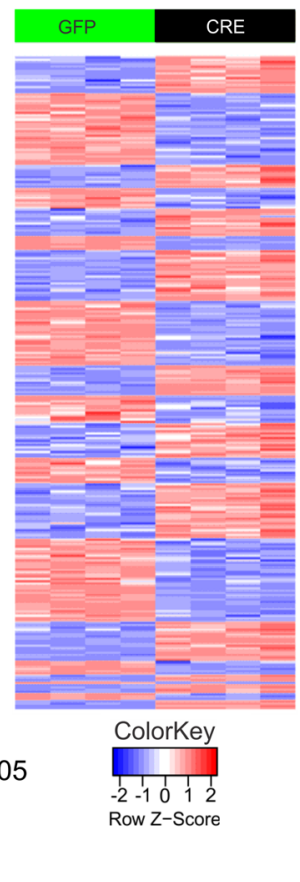

D

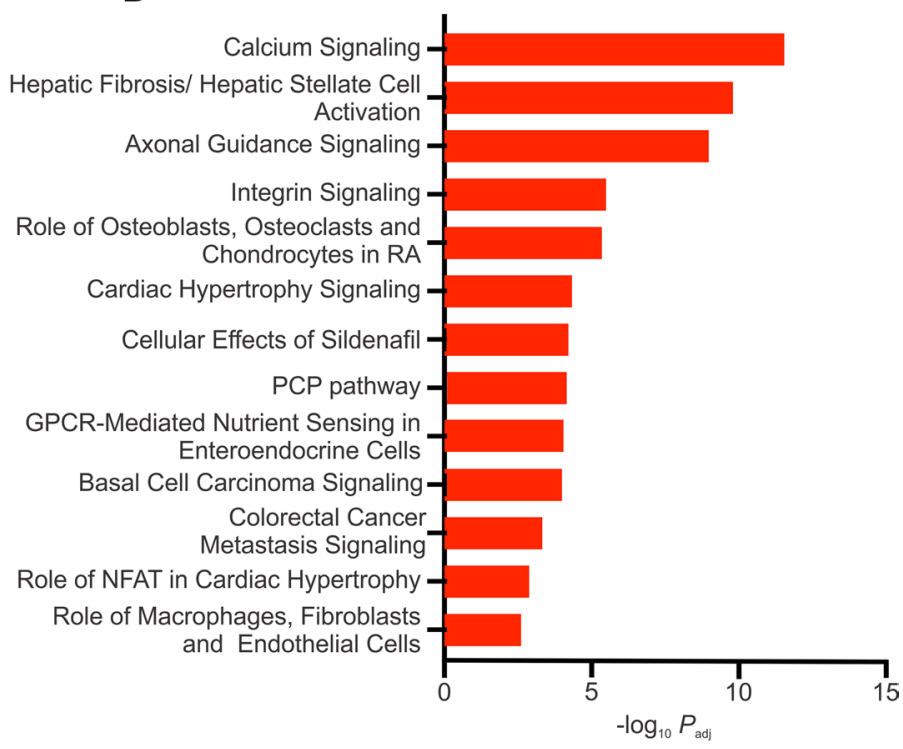

Figure 6. RNA-Seq of VICs infected with adenovirus expressing GFP or Cre. (A) Distribution of upregulated (Up) and downregulated (Down) differentially expressed genes 48 hours after viral infection. (B) Volcano plot of differentially expressed genes 48 hours after infection. Blue and red symbols indicate genes differentially expressed $\left(P_{a d j}<0.05\right)$ and $\log _{2}$-fold greater than 1.0 or less than -1.0 , respectively. Gray indicates genes differentially expressed $\left(P_{a d j}<0.05\right)$ and $\log _{2}$-fold less than 1.0 and greater than -1.0 . Black indicates genes not differentially expressed $\left(P_{a d j}>0.05\right)$. (C) Heat map of the 2724 transcripts for which expression in the Cre infected VICs was upregulated or downregulated by more than 2-fold. (D) Gene ontology analysis by IPA of upregulated genes. PCP, planar cell polarity; RA, rheumatoid arthritis

fibrosis/hepatic stellate cell-activation pathway $\left(P_{a d j}=5.68 \times 10^{-10}\right)$, also identified as the top gene set among upregulated transcripts in calcified versus normal human aortic valves (29). Processes such as increased collagen synthesis are key features of hepatic fibrosis and CAVD, and transcripts of 30 collagen genes were upregulated, 17 by more than 2-fold (Supplemental Table 1) after $\mathrm{Ca}_{\mathrm{v}} 1.2$ activation. Furthermore, the upregulation of collagen transcripts is another indicator of myofibroblast activation (30), as myofibroblasts lead to marked increases in collagen secretion. Moreover, IPA identified TGF- $\beta$ as the top upstream regulator of the differentially expressed genes, suggesting that initial $\mathrm{Ca}^{2+}$ influx in mouse VICs converges on activation of a similar set of genes as application of TGF- $\beta$, and that the resulting signaling in our mouse VIC cultures more closely represents a dystrophic rather than an osteogenic process (4), consistent with our culturing of the VICs on a hard plastic surface, known to promote dystrophic calcification, rather than on material like fibronectin that better promotes osteogenic calcification (4).

One component of the hepatic fibrosis/hepatic stellate cell-activation pathway, Agt, was the most highly upregulated gene (511-fold \pm 59-fold; $P_{a d j}<1 \times 10^{-300}$ ) in our data set. Agt encodes angiotensinogen, the precursor of angiotensin II and a key member of the renin-angiotensin-aldosterone system (RAAS), for which individual components are tightly regulated within several feed-forward and feedback loops. The transcript level is commonly used to quantify the abundance of angiotensinogen, which is almost immediately secreted from cells (31). $A G T$ is upregulated in human calcified aortic valves (29), local angiotensinogen synthesis and RAAS signaling drive aortic valve calcification (32-34), and proteomic analysis of calcified human aortic valves identified the angiotensin signaling as 1 of the upregulated pathways (35). Increased levels of the negative regulator of RAAS signaling, angiotensin-converting enzyme 2 (ACE2), is a biomarker for higher mortality rate and myocardial fibrosis in patients with CAVD (36). Therefore, we cataloged other RAAS signaling components (Supplemental Table 2), many of which are altered in CAVD, such as the Ace2, (Pro)renin receptor (Atp6ap2), and the angiotensin II receptor (Agtr1a), in addition to Agt.

While the VIC culture conditions favored dystrophic calcification over osteogenic calcification, 1 of the top signaling pathways identified by IPA analysis was the role of osteoblasts, osteoclasts, and chondrocytes in rheumatoid arthritis (Figure 6D), suggesting that $\mathrm{Ca}^{2+}$ influx through $\mathrm{Ca}_{\mathrm{v}} 1.2$ may be an activating signal 
for both calcification pathways. The enriched transcripts belonging to this set included multiple Wnt family members and their Frizzled receptors, as well as 3 of the 4 nuclear factor of activated T cells (Nfatc) genes (Nfatc1, Nfatc2, Nfatc4). In addition to being components of the gene set for the role of osteoblasts, osteoclasts, and chondrocytes in rheumatoid arthritis, the $\mathrm{Nfatc}$ gene products are $\mathrm{Ca}^{2+}$-responsive transcriptional regulators and thus serve as components of sensors capable of translating $\mathrm{Ca}^{2+}$ influx through $\mathrm{Ca}_{\mathrm{v}} 1.2$ to downstream $\mathrm{Ca}^{2+}$-signaling gene expression pathways (37). Together, the data from the VICs demonstrate early consequences of increased $\mathrm{Ca}^{2+}$ influx through $\mathrm{Ca}_{\mathrm{v}} 1.2$ in VICs.

\section{Discussion}

Building upon a study showing an association between increased expression of $\mathrm{Ca}_{\mathrm{v}} 1.2$ in the aortic valve and CAVD (19), our combination of VIC culture and in vivo models demonstrates that increased $\mathrm{Ca}^{2+}$ influx through $\mathrm{Ca}_{\mathrm{v}} 1.2$ is, indeed, causal for the initiation of calcific lesions within the aortic valve. The location of the lesions in the hinge regions in young mice maintained on normal chow rather than a high-cholesterol diet suggests that this is a relevant model for the early aortic valve lesions described in presymptomatic individuals (10). The role of $\mathrm{Ca}_{\mathrm{v}} 1.2$ in this model, echoing the GWAS of patients with more advanced CAVD (19), is underlined by our experiments showing that blocking $\mathrm{Ca}_{\mathrm{v}} 1$.2-channel activity with verapamil reduced the $\alpha$-SMA signal of myofibroblast activation in cultured VICs and decreased the valve lesions in our in vivo model. CAVD arises from myofibroblast differentiation of VICs in response to paracrine action of stimuli TGF- $\beta$, and our data show that $\mathrm{Ca}^{2+}$ influx through $\mathrm{Ca}_{\mathrm{v}} 1.2$ may be a key downstream step, because our in vivo $\mathrm{Ca}_{\mathrm{v}} 1.2$ mouse models showed lesion development in the absence of any such exogenous paracrine stimuli and in the absence of the hypercholesterolemia stimulus commonly used in CAVD mouse models.

The drivers for increased $\mathrm{Ca}_{\mathrm{v}} 1.2$ expression in diseased valves are not known, but the subsequent influx of $\mathrm{Ca}^{2+}$ through $\mathrm{Ca}_{\mathrm{v}} 1.2$ then is clearly poised to drive gene expression pathways that promote valve calcification, such as the marked upregulation of multiple collagen genes (Supplemental Table 1) or the activation of local RAAS signaling (Supplemental Table 2) that is known to promote valve calcification and may also be a promising target, based on a retrospective analysis (38).

That verapamil was effective in cultured VICs suggests the ability of $\mathrm{Ca}_{\mathrm{v}} 1.2 \mathrm{Ca}^{2+}$ channel antagonists to reduce myofibroblast activation is independent of an effect on blood pressure. While these agents may not be recommended for individuals with severely advanced disease (39), in part because $\mathrm{Ca}^{2+}$ channel antagonists and other agents that reduce afterload are generally contraindicated in severe aortic valve disease, $\mathrm{Ca}^{2+}$ channel antagonists could be well tolerated earlier in the disease process, such as when aortic sclerosis is recognized by echocardiography. The similarity between the location and morphology of these lesions and those found in presymptomatic individuals (10) underscores the translational potential of $\mathrm{Ca}^{2+}$ channel antagonists for early CAVD. Although dihydropyridines, which have less negative inotropy, may be the $\mathrm{Ca}^{2+}$ channel antagonist of choice, we were unable to assess them in our transgenic models, because of a designed dihydropyridine-resistance mutation to allow pharmacological distinction of the transgenic $\mathrm{Ca}_{\mathrm{v}} 1.2$ channels from endogenous channels (28). Moreover, because critical steps leading to CAVD, such as dystrophic calcification and osteogenesis, mimic processes identified in atherosclerotic calcification (40, 41), the efficacy of a $\mathrm{Ca}^{2+}$ channel antagonist in the VIC cultures resonates with previous clinical trial data (e.g., PREVENT, CAMELOT, ACCOMPLISH) showing that $\mathrm{Ca}_{\mathrm{v}} 1.2 \mathrm{Ca}^{2+}$ channel antagonists reduced cardiovascular events and caused regression of atherosclerotic plaques (42-44), which is especially notable because the CAMELOT trial researchers observed a benefit with amlodipine in patients with normal blood pressure. Despite the parallels between CAVD and atherosclerosis, pharmacological treatments that have been successful for atherosclerosis failed for CAVD (e.g., lipid lowering with statins) (45-48), emphasizing the need for the identification of new targets and the development of novel therapies for CAVD by uncovering disease mechanisms. $\mathrm{Ca}_{\mathrm{v}} 1.2 \mathrm{Ca}^{2+}$ channel antagonists may offer 1 such therapy that, to our knowledge, has not yet been investigated.

The RNA-Seq data obtained from cultured VICs provide several clues to important mediators downstream of $\mathrm{Ca}_{\mathrm{v}} 1.2$ and suggestions for additional therapeutic interventions and disease mechanisms. For example, Supplemental Table 3 lists key upregulated, $\mathrm{Ca}^{2+}$-sensitive signaling genes, including those encoding $\mathrm{Ca}^{2+}$ /calmodulin kinases (Camk2a and Camkk1), the NFATc components of the $\mathrm{Ca}^{2+}$-sensitive phosphatase calcineurin complex that mediate $\mathrm{Ca}^{2+}$-dependent transcription ( Nfatc1, Nfatc2, Nfatc4), and the $\mathrm{Ca}^{2+}$-binding second messenger and transcriptional regulator calreticulin (Calr), with $N f a t c 2$ being the top 
upregulated transcript. Thus, targeting NFAT-dependent transcription with inhibitors such as cyclosporine or tacrolimus may offer opportunities to prevent or delay progression of CAVD beyond the suggested efficacy of $\mathrm{Ca}_{\mathrm{v}} 1.2 \mathrm{Ca}^{2+}$ channel antagonists such as verapamil that was effective in our mouse model. Antagonism of the calcineurin-signaling pathway prevents graft atherosclerosis (49), suggesting an additional parallel between mechanisms driving atherosclerosis and CAVD.

Another example of a gene upregulated by $\mathrm{Ca}^{2+}$ influx through $\mathrm{Ca}_{\mathrm{v}} 1.2$ that provides potential mechanistic insight is $\operatorname{Ttr}\left(16.47\right.$-fold \pm 7.73 -fold; $\left.P_{a d j}=3.05 \times 10^{-6}\right)$. Ttr encodes transthyretin, a protein that deposits in the heart and cause cardiac amyloidosis. There are 2 types of cardiac amyloidosis, hereditary transthyretin cardiac amyloidosis that results from mutations in TTR, and senile transthyretin cardiac amyloidosis, in which WT transthyretin deposits in the myocardium and valves. Deposition of transthyretin is increasingly recognized in valves excised from patients undergoing valve replacement for CAVD (50), and cardiac amyloidosis is increasingly recognized as a comorbidity of CAVD (51). How WT transthyretin leads to amyloid deposition in senile cardiac amyloidosis is unclear, but a transgenic mouse model with TTR overexpression demonstrated amyloid deposition (52). Thus, the marked upregulation of Ttr in the VICs may provide a mechanism.

The mechanisms by which $\mathrm{Ca}_{\mathrm{v}} 1.2$, a voltage-gated $\mathrm{Ca}^{2+}$ channel that is usually considered in the context of excitable cells such as neurons or cardiomyocytes, is regulated in fibroblast-like VICs is not clear; membrane voltage in VICs has not been actively studied. Nevertheless, there is growing evidence that $\mathrm{Ca}_{\mathrm{v}} 1.2$ confers important functions in nonexcitable cells. Chondrocytes and osteoblasts appear to be prime examples, as others and we have shown $(21-23,27)$. Moreover, individuals with Timothy syndrome with the $\mathrm{Ca}_{\mathrm{v}} 1.2 \mathrm{G} 406 \mathrm{R}$ gain-of-function mutation suffer from various phenotypes in multiple nonexcitable tissues $(26,53)$, although these individuals have not been reported to have CAVD, likely because most known patients have short life spans. For the aortic valve, which is subject to mechanical shear stress, 1 possible means of further activating $\mathrm{Ca}_{\mathrm{v}} 1.2$ in vivo may be via activation of mechanosensitive channels such as Piezo1 that have been found in bone. Osteocytes express high levels of Piezo1 in static conditions and the Piezo1 transcripts increase with shear stress $(54,55)$. Stretch and mechanical forces cause Piezo1 or its homolog Piezo2 to open, providing an inward depolarizing current, including $\mathrm{Ca}^{2+}(56)$. Shear stress is a potent driver of the CAVD pathway (57) and, in our VIC cultures, Piezo1 is highly expressed: it is in the top $0.02 \%$ of all protein coding transcripts by read abundance.

In conclusion, our RNA-Seq data from VICs in which increased $\mathrm{Ca}^{2+}$ influx through $\mathrm{Ca}_{\mathrm{v}} 1.2$ identified multiple differentially expressed genes and signaling pathways common in CAVD, show that the association between $C A C N A 1 C$ SNPs and increased $\mathrm{Ca}^{2+}$ influx through $\mathrm{Ca}_{\mathrm{v}} 1.2$ with CAVD is, indeed, causal. Moreover, these collected data provide mechanistic insight. Our in vivo mouse-model studies showed increased $\mathrm{Ca}^{2+}$ influx through $\mathrm{Ca}_{\mathrm{v}} 1.2$ in the aortic valve resulted in lesion formation with dystrophic, chondrogenic, and osteogenic characteristics, reflecting later signaling events, which we hypothesize are driven by the initial cascade of gene expression changes observed in the cultures. An important result from these studies is that, because $\mathrm{Ca}_{\mathrm{v}} 1.2$ is druggable, targeted treatment with commonly used $\mathrm{Ca}^{2+}$ channel antagonists, if applied early in the disease process, may offer a therapeutic strategy, as we showed with verapamil. Our RNA-Seq data sets and these newly developed models suggest that further definition of the signaling events from $\mathrm{Ca}^{2+}$ influx through $\mathrm{Ca}_{\mathrm{v}} 1.2$ to myofibroblast activation and resultant valve calcification may reveal additional therapeutic targets for development.

\section{Methods}

Human aortic valve immunofluorescence. Immunofluorescent staining of human tissue samples was performed as previously described (58). Briefly, OCT-embedded (Tissue-Tek) frozen sections ( $7 \mu \mathrm{m})$ from control valves (obtained for heart transplant surgery) or calcified valves removed during surgical aortic valve replacement were fixed in an acetone/methanol solution for 10 minutes at $4^{\circ} \mathrm{C}$. After blocking for 1 hour, the sections were incubated with a custom-made polyclonal anti- $\alpha_{1 \mathrm{C}}\left(\mathrm{Ca}_{\mathrm{v}} 1.2\right)$ antibody (Yenzym, 1:50 dilution) (59-61) and a monoclonal anti-Runx2 antibody (Abnova, H00000860-M05).

$R N A$-Seq and bioinformatics. For the 48-hour data sets ( $n=4$ mice each) and the 72-hour data sets $(n=3$ mice each), library preparation and sequencing were performed at Weill Cornell Medicine Genomic Core Facility. The sequencing libraries were sequenced with single-end 50 bps on a HiSeq 4000 sequencer (Illumina). The raw sequencing reads in binary base call format were processed through bcl2fastq 2.19 (Illumina) for FASTQ conversion and demultiplexing. RNA reads were aligned and mapped to the GRCm38 mouse reference genome 
by STAR (version 2.5.2; https://github.com/alexdobin/STAR) (62), and transcriptome reconstruction was performed by Cufflinks (version 2.1.1; http://cole-trapnell-lab.github.io/cufflinks/). The abundance of transcripts was measured with Cufflinks in fragments per kilobase of exon model per million mapped reads (63, 64). Gene expression profiles were constructed for differential expression, cluster, and principle component analyses with the DESeq2 package (https://bioconductor.org/packages/release/bioc/html/DESeq2.html) (65), eliminating genes with insufficient base mean reads. For the canonical pathway and gene network analysis, data were analyzed via IPA (QIAGEN). Raw data are deposited in the Gene Expression Omnibus (GEO GSE151520).

$q P C R$. RNA was isolated from cultured mouse VICs using the RNeasy Mini Kit (QIAGEN) according to the manufacturer's instructions. Reverse transcription was performed using iScript cDNA Synthesis Kit (Bio-Rad). qPCR was performed in duplicate for each sample with QuantStudio 3 (Applied Biosystems) using SYBR green-based detection chemistries (Bio-Rad). Relative expression was quantified using the $\mathrm{Ct}$ method, except in Figure 5A, in which the raw Ct values are reported to emphasize the almost complete lack of valvular endothelial cell markers. GAPDH was used for a reference gene. For primer sequences used for qPCR, see Supplemental Table 4 (66-68).

Animals. C57BL/6J mice (B6) (catalog 00064) and $\mathrm{Ca}_{\mathrm{v}} 1.2^{\text {lacz/+ }}$ reporter mice (catalog 0005783) were purchased from Jackson Laboratory. ScxCre mouse (69) was provided by Ronen Schweitzer (Oregon Health and Science University, Portland, Oregon, USA). STOP-floxed WT or a G406R mutant $C a_{V} 1.2^{W T /+}$ and $C a_{V} 1.2^{T S /+}$ mice were described previously (28). Data from male and female mice were analyzed after initial observations did not reveal a sex difference.

Aortic VIC isolation and in vitro assays. Mouse primary aortic VICs were isolated as described previously (70). Briefly, aortic valves were dissected and pooled together $(n=3-5)$ and digested with collagenase to eliminate valve endothelial cells (VECs) and isolate VICs. Cells from passages 3-8 were used. Adenovirus expressing Cre recombinase (Vector Biolabs, 1045) or GFP (Vector Biolabs, 1060) were used to infect cells. For the verapamil assay, $10 \mu \mathrm{M}$ verapamil was added to $\mathrm{Ca}_{\mathrm{v}} 1.2^{\mathrm{TS}}$ cells in addition to adenovirus expressing either Cre recombinase or GFP. After 2 days of incubation, RNA was isolated and qPCR was performed.

Histology, pentachrome staining, and immunofluorescent staining. ScxCre;Ca $1.2^{T S /+}$ and ScxCre;Ca $1.2^{W T /+}$ mice and their control littermates $\left(C a_{V} 1.2^{T S /+}\right.$ and $\left.C a_{V} 1.2^{W T /+}\right)$ were sacrificed. Hearts were removed and fixed in $4 \%$ paraformaldehyde for 1 hour at room temperature. Tissue was further dehydrated through ethanol gradient washes, embedded in paraffin, and sectioned at $10 \mu \mathrm{m}$. Movat's pentachrome staining was performed according to the manufacturer's protocol (American MasterTech Scientific). For Aggrecan (1:500; Millipore, AB1031) and Sox9 (1:500; Millipore, AB5535) staining, tissues were treated with chondroitinase (200 mU/mL; Sigma) before blocking. For Runx2 (1:100; Novus Biologicals, NBP1-77472) and $\alpha$-SMA (1:200; Abcam, ab5694), antigen retrieval was performed using sodium citrate (10 mM, pH 6) before blocking.

Determination of calcium deposition in aortic valve. Hearts from $S c x C r e ; C a_{V} 1.2^{T S /+}$ and $S c x C r e ; C a_{V} 1.2^{W T /+}$ mice and their control littermates $\left(C a_{V} 1.2^{T S /+}\right.$ and $\left.C a_{V} 1.2^{W T /+}\right)$ were fixed in $4 \%$ paraformaldehyde, embedded in paraffin, and sectioned at $10 \mu \mathrm{m}$. For von Kossa staining to detect calcium deposition in the aortic valve (71), sections were incubated in $1 \%$ aqueous silver nitrate solution for 20 minutes in the UV chamber (Fisher Scientific, FB-UVXL-1000). Slides were rinsed in water, excess silver was removed with 5\% sodium thiosulfate for 5 minutes, and counterstained with nuclear fast red. For alizarin red staining, sections were fixed in $4 \%$ paraformaldehyde and incubated in alizarin red solution for 30 minutes.

Mini-osmotic pump implantation. A mini-osmotic pump (Alzet, 2004) filled with either vehicle (DMSO diluted 1:1 in water) or verapamil solution $(10 \mathrm{mg} / \mathrm{kg} / \mathrm{d})$ was implanted s.c. for infusion at a rate of $10 \mathrm{mg} /$ $\mathrm{kg} / \mathrm{d}$. Incision was closed with a surgical stapler. After 28 days of drug delivery through a mini osmotic pump, mice were sacrificed and the heart samples were processed for histology.

Statistics. Data analysis was performed using GraphPad Prism, version 8.4. All averaged data are presented as mean \pm SEM. Statistical significance was determined using 2-tailed $t$ tests, a 2-way ANOVA with post hoc correction for multiple comparisons, or $\chi^{2}$ test. $P<0.05$ was considered statistically significant. For RNA-Seq differential expression analyses, pairwise comparisons between 2 or more groups were made using parametric tests in which read counts follow a negative binomial distribution with a gene-specific dispersion parameter. $P_{a d j}$ values were calculated on the basis of the Benjamin-Hochberg method to adjust for multiple testing.

Study approval. Animals were handled according to NIH Guide for the Care and Use of Laboratory Animals. The research was approved by the Weill Cornell Research Animal Resource Center. 
Human aortic valve samples were obtained from the valve replacement and heart transplantation surgeries at Mount Sinai Hospital, New York, New York. The study was approved (GCO 13-1821/HS 13-00741) by the IRB of the Icahn School of Medicine at Mount Sinai. All patients gave written informed consent prior to surgery.

\section{Author contributions}

$\mathrm{MM}, \mathrm{RB}, \mathrm{SOM}$, and GSP designed the research studies. MM and RB conducted experiments, acquired data, analyzed data. MS, YH, and AG conducted experiments, acquired data, and analyzed data. MM, SOM, and GSP wrote the manuscript.

\section{Acknowledgments}

Supported by NIH grants R01HD090132 and R01 R01HL151190 and a Harrington Discovery Institute Scholar-Innovator Award (to GSP); NIH grant R01HL146149 (to SOM and GSP); American Heart Association Postdoctoral Fellowship 17POST33670699 and Ehrenkranz Young Scientist Award (to MM).

Address correspondence to: Geoffrey S. Pitt, Cardiovascular Research Institute, Weill Cornell Medicine, 413 E. 69th Street, Belfer Building 502, New York, New York 10021, USA. Phone: 646.962.7641; Email: geoffrey.pitt@med.cornell.edu.

1. Nishimura RA, et al. 2014 AHA/ACC guideline for the management of patients with valvular heart disease: a report of the American College of Cardiology/American Heart Association task force on practice guidelines. Circulation. 2014;129(23):e521-e643.

2. Lindman BR, Merryman WD. Unloading the stenotic path to identifying medical therapy for calcific aortic valve disease: barriers and opportunities. Circulation. 2021;143(15):1455-1457.

3. Akat K, et al. Aortic valve calcification: basic science to clinical practice. Heart. 2009;95(8):616-623.

4. Rutkovskiy A, et al. Valve interstitial cells: the key to understanding the pathophysiology of heart valve calcification. $J A m$ Heart Assoc. 2017;6(9):e006339.

5. Rajamannan NM, et al. Human aortic valve calcification is associated with an osteoblast phenotype. Circulation. 2003;107(17):2181-2184.

6. Wirrig EE, et al. Differential expression of cartilage and bone-related proteins in pediatric and adult diseased aortic valves. J Mol Cell Cardiol. 2011;50(3):561-569.

7. Alfieri CM, et al. Wnt signaling in heart valve development and osteogenic gene induction. Dev Biol. 2010;338(2):127-135.

8. Gomez-Stallons MV, et al. Bone morphogenetic protein signaling is required for aortic valve calcification. Arterioscler Thromb Vasc Biol. 2016;36(7):1398-1405

9. Fang M, et al. Loss of $\beta$-catenin promotes chondrogenic differentiation of aortic valve interstitial cells. Arterioscler Thromb Vasc Biol. 2014;34(12):2601-2608.

10. Gomez-Stallons MV, et al. Calcification and extracellular matrix dysregulation in human postmortem and surgical aortic valves Heart. 2019;105(21):1616-1621.

11. Thanassoulis G, et al. Genetic associations with valvular calcification and aortic stenosis. N Engl J Med. 2013;368(6):503-512.

12. Stewart BF, et al. Clinical factors associated with calcific aortic valve disease. Cardiovascular health study. J Am Coll Cardiol. 1997;29(3):630-634.

13. Gotoh T, et al. Correlation between lipoprotein(a) and aortic valve sclerosis assessed by echocardiography (the JMS cardiac echo and cohort study). Am J Cardiol. 1995;76(12):928-932.

14. Glader CA, et al. Lipoprotein(a), Chlamydia pneumoniae, leptin and tissue plasminogen activator as risk markers for valvular aortic stenosis. Eur Heart J. 2003;24(2):198-208.

15. Bozbas H, et al. Effects of serum levels of novel atherosclerotic risk factors on aortic valve calcification. J Heart Valve Dis. 2007;16(4):387-393.

16. Helgadottir A, et al. Genome-wide analysis yields new loci associating with aortic valve stenosis. Nat Commun. $2018 ; 9(1): 987$.

17. Theriault S, et al. A transcriptome-wide association study identifies PALMD as a susceptibility gene for calcific aortic valve stenosis. Nat Commun. 2018;9(1):988.

18. Theriault S, et al. Genetic association analyses highlight IL6, ALPL, and NAV1 as 3 new susceptibility genes underlying calcific aortic valve stenosis. Circ Genom Precis Med. 2019;12(10):e002617.

19. Guauque-Olarte S, et al. Calcium signaling pathway genes RUNX2 and CACNA1C are associated with calcific aortic valve disease. Circ Cardiovasc Genet. 2015;8(6):812-822.

20. Pitt GS, et al. Voltage-gated calcium channels in nonexcitable tissues. Annu Rev Physiol. 2021;83:183-203.

21. Ramachandran KV, et al. Calcium influx through L-type CaV1.2 Ca2+ channels regulates mandibular development. J Clin Invest. 2013;123(4):1638-1646.

22. Cao C, et al. The $\mathrm{Ca}_{\mathrm{v}} 1.2$ L-type calcium channel regulates bone homeostasis in the middle and inner ear. Bone. 2019;125:160-168.

23. Cao $\mathrm{C}$, et al. Increased $\mathrm{Ca} 2+$ signaling through $\mathrm{CaV} 1.2$ promotes bone formation and prevents estrogen deficiency-induced bone loss. JCI Insight. 2017;2(22):e95512.

24. Lincoln J, et al. Development of heart valve leaflets and supporting apparatus in chicken and mouse embryos. Dev Dyn. $2004 ; 230(2): 239-250$. 
25. Levay AK, et al. Scleraxis is required for cell lineage differentiation and extracellular matrix remodeling during murine heart valve formation in vivo. Circ Res. 2008;103(9):948-956.

26. Splawski I, et al. Ca(V)1.2 calcium channel dysfunction causes a multisystem disorder including arrhythmia and autism. Cell. 2004;119(1):19-31.

27. Atsuta Y, et al. L-type voltage-gated $\mathrm{Ca}^{2+}$ channel CaV1.2 regulates chondrogenesis during limb development. Proc Natl Acad US A. 2019;116(43):21592-21601.

28. Pasca SP, et al. Using iPSC-derived neurons to uncover cellular phenotypes associated with Timothy syndrome. Nat Med. 2011;17(12):1657-1662.

29. Guauque-Olarte S, et al. RNA expression profile of calcified bicuspid, tricuspid, and normal human aortic valves by RNA sequencing. Physiol Genomics. 2016;48(10):749-761.

30. Baum J, Duffy HS. Fibroblasts and myofibroblasts: what are we talking about? J Cardiovasc Pharmacol. 2011;57(4):376-379.

31. Wu C, et al. Molecular and pathophysiological features of angiotensinogen: a mini review. N Am J Med Sci (Boston). 2011;4(4):183-190.

32. O'Brien $\mathrm{KD}$, et al. Association of angiotensin-converting enzyme with low-density lipoprotein in aortic valvular lesions and in human plasma. Circulation. 2002;106(17):2224-2230.

33. Helske S, et al. Induction of local angiotensin II-producing systems in stenotic aortic valves. J Am Coll Cardiol. 2004;44(9):1859-1866

34. Peltonen T, et al. (Pro)renin receptors and angiotensin converting enzyme 2/angiotensin-(1-7)/Mas receptor axis in human aortic valve stenosis. Atherosclerosis. 2011;216(1):35-43.

35. Schlotter F, et al. Spatiotemporal multi-omics mapping generates a molecular atlas of the aortic valve and reveals networks driving disease. Circulation. 2018;138(4):377-393.

36. Ramchand J, et al. Plasma ACE2 activity predicts mortality in aortic stenosis and is associated with severe myocardial fibrosis. JACC Cardiovasc Imaging. 2020;13(3):655-664.

37. Graef IA, et al. L-type calcium channels and GSK-3 regulate the activity of NF-ATc4 in hippocampal neurons. Nature. 1999;401(6754):703-708.

38. Nadir MA, et al. Impact of renin-angiotensin system blockade therapy on outcome in aortic stenosis. J Am Coll Cardiol. 2011;58(6):570-576.

39. Saeed S, et al. Antihypertensive treatment with calcium channel blockers in patients with moderate or severe aortic stenosis: Relationship with all-cause mortality. Int J Cardiol. 2020;298:122-125.

40. Doherty TM, et al. Calcification in atherosclerosis: bone biology and chronic inflammation at the arterial crossroads. Proc Natl Acad Sci U S A. 2003;100(20):11201-11206.

41. Hjortnaes J, et al. Arterial and aortic valve calcification inversely correlates with osteoporotic bone remodelling: a role for inflammation. Eur Heart J. 2010;31(16):1975-1984.

42. Pitt B, et al. Effect of amlodipine on the progression of atherosclerosis and the occurrence of clinical events. PREVENT Investigators. Circulation. 2000;102(13):1503-1510.

43. Nissen SE, et al. Effect of antihypertensive agents on cardiovascular events in patients with coronary disease and normal blood pressure: the CAMELOT study: a randomized controlled trial. JAMA. 2004;292(18):2217-2225.

44. Weber MA, et al. Effects of body size and hypertension treatments on cardiovascular event rates: subanalysis of the ACCOMPLISH randomised controlled trial. Lancet. 2013;381(9866):537-545.

45. Rossebo AB, et al. Intensive lipid lowering with simvastatin and ezetimibe in aortic stenosis. NEngl J Med. 2008;359(13):1343-1356.

46. Cowell SJ, et al. A randomized trial of intensive lipid-lowering therapy in calcific aortic stenosis. NEngl J Med. 2005;352(23):2389-2397.

47. Moura LM, et al. Rosuvastatin affecting aortic valve endothelium to slow the progression of aortic stenosis. J Am Coll Cardiol. 2007;49(5):554-561.

48. Chan KL, et al. Effect of Lipid lowering with rosuvastatin on progression of aortic stenosis: results of the aortic stenosis progression observation: measuring effects of rosuvastatin (ASTRONOMER) trial. Circulation. 2010;121(2):306-314

49. Lindenfeld J, et al. Drug therapy in the heart transplant recipient: part II: immunosuppressive drugs. Circulation. 2004;110(25):3858-3865.

50. Kristen AV, et al. High prevalence of amyloid in 150 surgically removed heart valves--a comparison of histological and clinical data reveals a correlation to atheroinflammatory conditions. Cardiovasc Pathol. 2010;19(4):228-235.

51. Ternacle J, et al. Aortic stenosis and cardiac amyloidosis: JACC review topic of the week. J Am Coll Cardiol. 2019;74(21):2638-2651.

52. Buxbaum JN, et al. Why are some amyloidoses systemic? Does hepatic "chaperoning at a distance" prevent cardiac deposition in a transgenic model of human senile systemic (transthyretin) amyloidosis? FASEB J. 2012;26(6):2283-2293

53. Splawski I, et al. Severe arrhythmia disorder caused by cardiac L-type calcium channel mutations. Proc Natl Acad Sci U S A 2005;102(23):8089-8096.

54. Sun W, et al. The mechanosensitive Piezo1 channel is required for bone formation. Elife. 2019;8:e47454

55. Li X, et al. Stimulation of Piezo1 by mechanical signals promotes bone anabolism. Elife. 2019;8:e49631.

56. Wu J, et al. Touch, tension, and transduction — the function and regulation of piezo ion channels. Trends Biochem Sci. 2017;42(1):57-71

57. Back M, et al. Biomechanical factors in the biology of aortic wall and aortic valve diseases. Cardiovasc Res. 2013;99(2):232-241.

58. Bouchareb R, et al. Proteomic architecture of valvular extracellular matrix: FNDC1 and MXRA5 are new biomarkers of aortic stenosis. JACC Basic Transl Sci. 2021;6(1):25-39.

59. Yang L, et al. Protein kinase C isoforms differentially phosphorylate Ca(v)1.2 alpha(1c). Biochemistry. 2009;48(28):6674-6683.

60. Yang L, et al. Cardiac L-type calcium channel (Cav1.2) associates with gamma subunits. FASEB J. 2011;25(3):928-936.

61. Liu G, et al. Mechanism of adrenergic $\mathrm{Ca}_{\mathrm{v}} 1.2$ stimulation revealed by proximity proteomics. Nature. 2020;577(7792):695-700

62. Dobin A, et al. STAR: ultrafast universal RNA-seq aligner. Bioinformatics. 2013;29(1):15-21.

63. Trapnell C, et al. Transcript assembly and quantification by RNA-Seq reveals unannotated transcripts and isoform switching during cell differentiation. Nat Biotechnol. 2010;28(5):511-515.

64. Trapnell C, et al. Differential analysis of gene regulation at transcript resolution with RNA-seq. Nat Biotechnol. 2013;31(1):46-53. 
65. Love MI, et al. Moderated estimation of fold change and dispersion for RNA-seq data with DESeq2. Genome Biol. 2014;15(12):550.

66. Spandidos A, et al. A comprehensive collection of experimentally validated primers for polymerase chain reaction quantitation of murine transcript abundance. BMC Genomics. 2008;9:633.

67. Spandidos A, et al. PrimerBank: a resource of human and mouse PCR primer pairs for gene expression detection and quantification. Nucleic Acids Res. 2010;38(database issue):D792-D799.

68. Wang XW, Seed B. A PCR primer bank for quantitative gene expression analysis. Nucleic Acids Res. 2003;31(24):e154.

69. Blitz E, et al. Bone ridge patterning during musculoskeletal assembly is mediated through SCX regulation of Bmp4 at the tendon-skeleton junction. Dev Cell. 2009;17(6):861-873.

70. E1 Husseini D, et al. P2Y2 receptor represses IL-6 expression by valve interstitial cells through Akt: implication for calcific aortic valve disease. J Mol Cell Cardiol. 2014;72:146-156.

71. Drury RAB. Theory and practice of histotechnology. J Clin Pathol. 1981;34(12):1406. 